\title{
Retrorectal Mucinous Adenocarcinoma Arising from a Tailgut Cyst: A Case Report
}

\author{
Manon Baverez $^{\mathrm{a}} \quad$ Emilie Thibaudeau $^{\mathrm{b}}$ Vincent Libois $^{c}$ Olivier Kerdraon ${ }^{d}$ \\ Hélène Senellart ${ }^{a}$ Jean-Luc Raoul ${ }^{a}$ \\ aDepartment of Medical Oncology, Institut de Cancérologie de l'Ouest, Saint-Herblain, \\ France; 'bepartment of Surgical Oncology, Institut de Cancérologie de I'Ouest, \\ Saint-Herblain, France; 'Department of Radiotherapy, Institut de Cancérologie de I'Ouest, \\ Saint-Herblain, France; ${ }^{d}$ Department of Pathology, Institut de Cancérologie de I'Ouest, \\ Saint-Herblain, France
}

\section{Keywords}

Retrorectal cyst · Tailgut cyst · Malignant transformation

\section{Abstract}

We report the case of a 57-year-old woman who presented with local invasion of the anal canal by mucinous adenocarcinoma, the malignant transformation of a long-term preexisting retrorectal tailgut cyst. This progression is infrequent and justifies preemptive surgical treatment of retrorectal cysts.

\section{Introduction}

Tailgut cysts (or retrorectal cystic hamartomas) are often described as congenital cysts arising in the retrorectal-presacral space due to remnants of the primitive tailgut. These rare cysts are more frequently observed in women and generally asymptomatic. Surgical treatment is necessary because of the risk of complications including recurrent perianal suppuration and malignant transformation [1]. The rate of malignant transformation is poorly understood but seems higher than initially estimated [2]; these are usually mucinous adenocarcinoma but can be transitional cell carcinoma, carcinoid tumors, or squamous cell cancers. We report the case of a 57-year-old woman who, perhaps for years, had been losing a small amount of mucoid fluid from a small sus-anal opening, and who experienced anal discomfort revealing retroanal mucinous adenocarcinoma invading her anal sphincter and developing on a tailgut cyst.

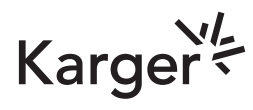




\section{Case Reports in Oncology}

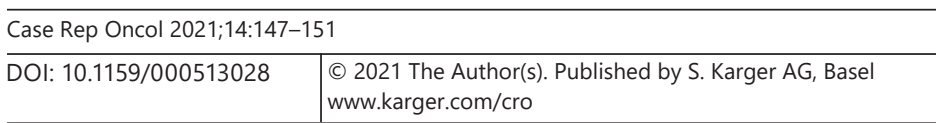

Baverez et al.: Malignant Transformation of a Tailgut Cyst
Fig. 1. CT scan showing a polycystic retroanal mass containing calcifications.

Fig. 2. Contrast-enhanced pelvic MRI, sagittal view: huge retroanal multilocular cystic mass invading the anal canal (arrow) and skin (arrowhead); the anterior margin was irregular, thickened, and poorly defined.
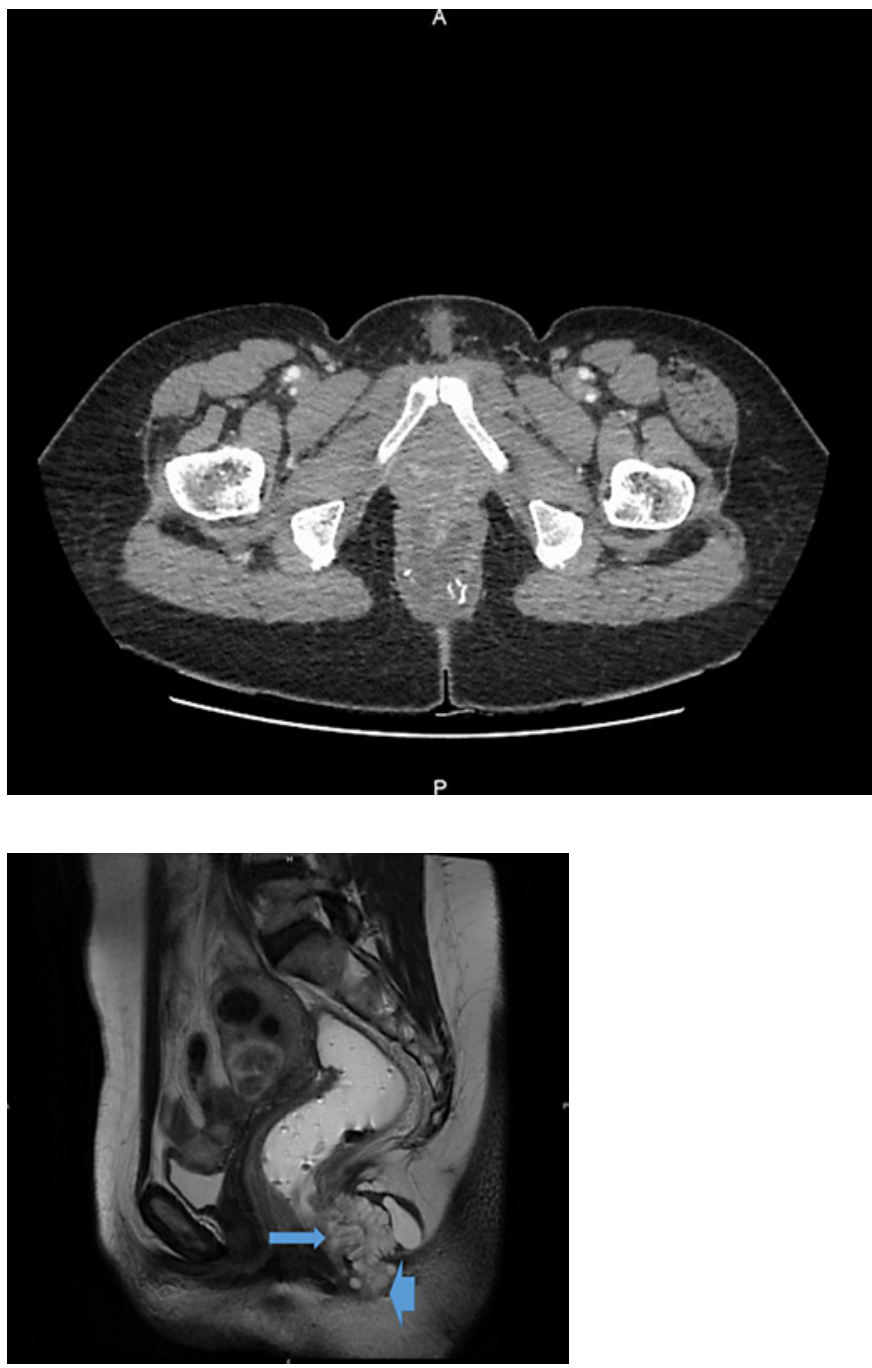

\section{Case}

At least 5 years ago, this 57-year-old woman from Mali noticed the loss of mucoid fluid from her perianal area with no pain or blood loss. Her husband had noticed a small opening (1-2 $\mathrm{mm}$ in diameter) located 2-3 cm above her anal verge far earlier. In 2017 she consulted the physician, who noticed and removed 3 jelly-like $10-\mathrm{mm}$ masses above her anal verge; no other exploration was performed or planned.

In 2018, her anal discomfort worsened and she again sought medical advice. On visual inspection, a 20-mm mass with ulcerations and mucoid areas was observed a few millimeters above her anal verge. On digital rectal examination, the physician found a hard mass of more than $40 \mathrm{~mm}$ invading the posterior part of the anal canal and destroying the anal sphincters. Biopsies revealed a villous tumor with areas of high-grade dysplasia and others of mucinous adenocarcinoma. A colonoscopy found no further lesions. A medical workup was then performed. On CT scan, she had a huge retroanal mass developed under the anal verge, pseudo-cystic, and partly calcified (Fig. 1); a large $(40 \times 25 \mathrm{~mm})$ mediastinal bronchogenic cyst was also seen. On MRI (Fig. 2, Fig. 3), a thin-walled, well-defined polycystic mass of 45 $\mathrm{mm}$ was observed but its anterior wall was ill defined, with signs of invasion of the anal sphincter and skin; the tumor was gadolinium enhanced. An ${ }^{18} \mathrm{~F}$ PET scan found no further 
Fig. 3. Contrast-enhanced pelvic MRI, axial view: invasion of the anal canal (arrowhead) and destruction of the anal sphincter by a retroanal polycystic mass (arrows).

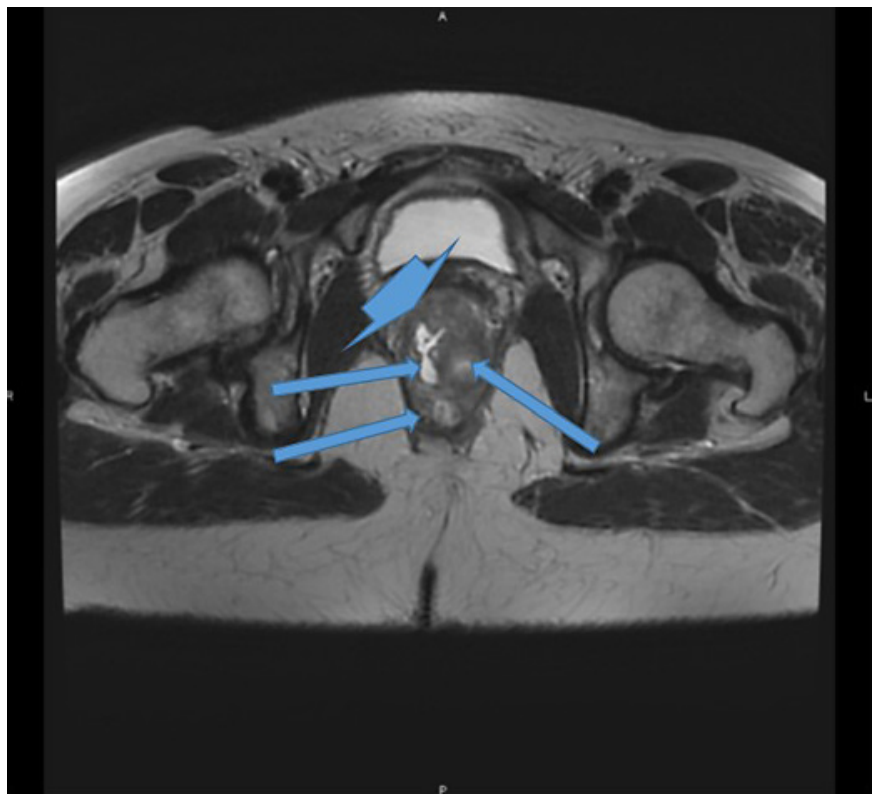

metabolic areas. The thoracic cyst was shown to be a bronchogenic cyst. On laboratory tests, CEA was elevated at $30 \mathrm{ng} / \mathrm{mL}$. The tumor was then diagnosed as a retrorectal cyst's malignant transformation into mucinous adenocarcinoma. After a multidisciplinary team meeting, an abdominoperineal resection following neoadjuvant chemoradiotherapy (50 Gy in 25 fractions plus concurrent capecitabine $800 \mathrm{mg} / \mathrm{m}^{2}$ twice daily) was decided. A second MRI was performed 2 months after completion of the neoadjuvant regimen and the images remained stable. A laparoscopic abdominoperineal resection was performed with no major problems. On pathological examination, a 55-mm mucinous lesion invading and destroying the posterior part of the anal region was described. Microscopically, the tumor was mucinous adenocarcinoma invading the external sphincter, anal canal, and perianal skin. None of the 24 examined lymph nodes was cancerous. The tumor was classified as ypT3ypN0. Biologically, postoperative CEA was normal $(1.2 \mathrm{ng} / \mathrm{mL})$. Two years after diagnosis, the patient is alive with no progressive disease.

\section{Discussion}

Tailgut cysts are a part of neoplastic retroperitoneal cystic masses separated from the abdominal and pelvic organs, including cystic lymphangioma, mucinous cystadenoma, cystic teratoma, cystic mesothelioma, Müllerian cyst, epidermoid cyst, and bronchogenic cyst [3]. Bronchogenic cysts, rare benign congenital anomalies originating from the embryonic foregut ventral segment, are sometimes found in a retroperitoneal location [3]. Our patient had both a bronchogenic cyst and a tailgut cyst.

In a recent series of 52 retrorectal tumors, $56 \%$ were tailgut cysts, and only 4 were malignant: one was a malignant tailgut cyst, one a GIST, one a leiomyosarcoma, and one an angiomyxoma [4].

In most cases, patients with tailgut cyst present with common symptoms such as constipation, obstructed defecation, dysuria, and urinary tract infection, all signs usually related to compression by the tumor mass. Histologically, these cysts may contain squamous, mucinous, or ciliated epithelium. 
On imaging, tailgut cysts and cystic lymphangioma are usually polycystic, while other presacral cystic masses (epidermoid cyst, dermoid cyst, rectal duplication cyst, anterior meningocele) are usually unilocular [5].

In cases of malignant transformation, imaging and particularly MRI is of major importance; transformation may be suspected on MRI in view of cystic nodular wall thickening [2], usually heterogeneous, with irregular margins, solid areas, and gadolinium enhancement [4]. Histologically, although adenocarcinomas are the most frequent histological types, neuroendocrine carcinomas, transitional cell carcinomas, sarcomas, or squamous cell carcinomas have also been observed and these different epithelia often coexist [2,6]. In our case, the retrorectal cyst had malignant degeneration mainly on the anterior wall and had invaded the anal sphincters and perianal skin. Other diagnoses are infrequently reported following such appearances on imaging. One case of rectal mucinous adenocarcinoma invading retrorectal dermoid cysts was recently reported [7]. Another very similar case of retrorectal mass, multilocular cystic on MRI with disseminated calcifications, involved retrorectal metastasis of pancreatic mucinous adenocarcinoma [8].

Treatment of such cancer arising from tailgut cysts is based on surgery guided by preoperative imaging. If imaging shows suspicious features, a biopsy is mandatory before proposing neoadjuvant treatment. If the tumor invades the rectum or skin, biopsy is simple. If not, the risks of biopsy are tumor spread, infection, and missing the transformed location. The surgical approach depends on tumor location. In cases like ours, neoadjuvant chemoradiotherapy similar to locally advanced rectal adenocarcinoma was given to decrease the risk of postoperative recurrence.

\section{Statement of Ethics}

Written consent for publication was obtained from the patient for publication of this case report and accompanying images.

\section{Conflict of Interest Statement}

All the authors declare that there is no conflict of interest that could be perceived as prejudicing the impartiality of the research reported.

\section{Funding Sources}

This research did not receive any specific grant from any funding agency in the public, commercial, or not-for-profit sector.

\section{Author Contributions}

All authors contributed to the care of the patient, participated in the writing of the paper, and accepted the final version of the manuscript.

\section{Karger'}




\section{References}

1 Hufkens AS, Cools P, Leyman P. Tailgut cyst: report of three cases and review of the literature. Acta Chir Belg. 2019;119(2):110-7.

2 Nicoll K, Bartrop C, Walsh S, Foster R, Duncan G, Payne C, et al. Malignant transformation of tailgut cysts is significantly higher than previously reported: systematic review of cases in the literature. Colorectal Dis. 2019;21(8):869-78.

3 Hegazi TM, Al-Sharydah AM, Lee KS, Mortele K. Retroperitoneal cystic masses: magnetic resonance imaging features. Abdom Radiol (NY). 2020;45(2):499-511.

4 Carpelan-Holmström M, Koskenvuo L, Haapamäki C, Renkonen-Sinisalo L, Lepistö A. Clinical management of 52 consecutive retro-rectal tumours treated at a tertiary referral centre. Colorectal Dis. 2020 Oct;22(10): 1279-85.

5 Yang DM, Park CH, Jin W, Chang SK, Kim JE, Choi SJ, et al. Tailgut cyst: MRI evaluation. AJR Am J Roentgenol. 2005;184(5):1519-23.

6 Liang F, Li J, Yu K, Zhang K, Liu T, Li J. Tailgut Cysts with Malignant Transformation: Features, Diagnosis, and Treatment. Med Sci Monit. 2020;26:e919803.

7 Wang R, Yan Z. Rectal Mucinous Adenocarcinoma Invading Retrorectal Dermoid Cysts: A Case Report. Front Oncol. 2019;9:1389.

8 Kondo T. Retrorectal tumour. The Lancet Oncology. 2018;19(11):e654. 